\title{
Geoestatística na determinação da variabilidade espacial de características químicas do solo sob diferentes preparos ${ }^{(1)}$
}

\author{
José Ruy Porto de Carvalho(2), Pedro Marques da Silveira ${ }^{(3)}$ e Sidney Rosa Vieira(4)
}

\begin{abstract}
Resumo - O objetivo deste trabalho foi estudar, mediante a geoestatística, a variabilidade espacial de $\mathrm{pH}, \mathrm{Ca}, \mathrm{Mg}, \mathrm{P}$ e K em Latossolo Vermelho-Escuro distrófico, textura argilosa, cultivado durante cinco anos consecutivos (1992-1996), em três sistemas de preparo (arado, grade e plantio direto) na Embrapa-Centro Nacional de Pesquisa de Arroz e Feijão, em Santo Antônio de Goiás, GO. Das 30 combinações entre características químicas do solo, profundidades de coleta e sistemas de preparo, 14 apresentaram efeito pepita puro, indicando ausência de dependência espacial. Semivariogramas direcionais revelaram forte e moderada dependência espacial na direção de Y. Experimentos longevos com práticas culturais orientadas em uma única direção tendem a mudar a estrutura espacial das propriedades do solo, o que indica ser a razão dos resultados obtidos. A direção de anisotropia está mais associada com o tratamento arado e a mais forte dependência espacial foi verificada com relação ao $\mathrm{pH}$ no sistema de preparo arado na profundidade de $5-20 \mathrm{~cm}$. A localização das amostras para estimar os valores das características químicas do solo deve levar em conta as operações de campo, e cuidados devem ser tomados em relação à amostragem casual. As amostras devem ser retiradas em outras direções, para que uma representação mais realista da área amostrada seja obtida.
\end{abstract}

Termos para indexação: pH do solo, preparo do solo, sistema de cultivo, métodos de análise.

\section{Geostatistics to determine spatial variability of soil chemical properties using different preparation systems}

\begin{abstract}
Spatial variability of $\mathrm{pH}, \mathrm{Ca}, \mathrm{Mg}, \mathrm{P}$ and $\mathrm{K}$ under three soil preparation systems (moldboard plough, harrow disc and no-tillage) was studied using geostatistical concepts in clayey Oxisol, in Santo Antônio de Goiás, GO, Brazil, at Embrapa-Centro Nacional de Pesquisa de Arroz e Feijão, for five consecutive years (1992-1996). Within a total of 30 combinations among soil chemical properties, soil depth and preparation system, 14 presented pure nugget effect, indicating absence of spatial dependence. Directional semivariograms revealed strong and moderate spatial dependence in the direction of Y. Experiments conducted for a long time and cultural practice conducted in a single direction tend to change the spatial structure of the soil properties. The anisotropy direction was more associated with harrow disc as a system of soil preparation and the strongest spatial dependence was verified for $\mathrm{pH}$ at $5-20 \mathrm{~cm}$ soil depth. These results suggest that sample location to estimate the values of the soil chemical properties should take field operations into account and should be carefully selected. Samples should be taken in other directions, so that a more realistic representation of the sampled area could be obtained.
\end{abstract}

Index terms: soil $\mathrm{pH}$, soil preparation, cropping systems, analytical methods.

(1) Aceito para publicação em 18 de dezembro de 2001 .

(2) Embrapa-Centro Nacional de Pesquisa Tecnológica em Informática para a Agricultura, Caixa Postal 6041, CEP 13083-970 Barão Geraldo, Campinas, SP. E-mail: jruy@cnptia.embrapa.br

(3) Embrapa-Centro Nacional de Pesquisa de Arroz e Feijão, Caixa Postal 179, CEP 75375-000 Santo Antônio de Goiás, GO. E-mail: pmarques@cnpaf.embrapa.br

(4) Instituto Agronômico, Centro de Solos e Recursos Agroambientais, Caixa Postal 28, CEP 13001-970 Campinas, SP. E-mail: sidney@iac.br

\section{Introdução}

Avanços tecnológicos na agropecuária têm mostrado a importância de se medir a variação espacial e temporal de propriedades que afetam o rendimento das culturas, com o objetivo de otimizar o aproveitamento de recursos e diminuir custos. O gerenciamento do processo de produção em função da variabilidade é o que se convencionou chamar Agricultura de Precisão. Seu objetivo é correlacionar cau- 
sas e efeitos a partir de séries históricas de dados e de sua distribuição espacial.

Cientistas da área de solos se preocupam com o problema de variação espacial e temporal dos solos desde o começo do século. Somente nas décadas de 50 e 60, com o avanço na teoria de estatística espacial, é que os cientistas começaram a perceber a potencialidade deste instrumento para o manuseio de dados quantitativos, facilitando a compreensão da variabilidade do solo (Burrough et al., 1994).

Na maioria dos campos do conhecimento científico, o objetivo é pesquisar características dos elementos de uma população. Uma vez definida a população objeto de estudo, necessita-se escolher a melhor maneira de estudar algumas de suas características. Na maioria das vezes, a população de interesse é demasiadamente grande, tornando-se impossível realizar um levantamento de dados de todos os seus elementos. Neste caso, deve-se delimitar as observações a uma amostra da população, a qual deve reproduzir, o mais fielmente possível, suas características (Carvalho, 1999).

Segundo Orlando Filho \& Rodella (1983), cerca de 80 a $85 \%$ do erro total nos resultados usados na recomendação de fertilizantes e corretivos podem ser atribuídos à amostragem no campo e de 15 a $20 \%$ podem ser decorrentes do trabalho de laboratório. Daí a necessidade de um bom plano amostral. A amostragem de solo é um dos procedimentos mais importantes, em qualquer programa de pesquisa na área, pois de nada valem análises químicas sofisticadas e extremamente rigorosas de suas variáveis, se as amostras coletadas não são representativas da área em estudo (Chung et al., 1995).

O objetivo deste trabalho foi estudar, por meio da Geoestatística, a variabilidade espacial de algumas características químicas do solo e avaliar seu comportamento sob três sistemas de preparo (arado, grade e plantio direto).

\section{Material e Métodos}

O experimento foi realizado na Fazenda Capivara, da Embrapa-Centro Nacional de Pesquisa de Arroz e Feijão, em Santo Antônio de Goiás, GO, em um Latossolo Vermelho-Escuro distrófico, textura argilosa, cultivado durante cinco anos (1992 a 1996), com as culturas de milho no verão e feijoeiro no inverno, sob irrigação por aspersão, sistema de pivô central. Essas duas espécies foram cultivadas sob três tratamentos de preparo do solo: arado de aiveca, grade aradora e plantio direto. No tratamento arado, foi usado arado de três aivecas, incorporando-se ao solo os resíduos das culturas até a profundidade de $30 \mathrm{~cm}$, seguido de uma gradagem com grade destorroadora. $\mathrm{Na}$ aração com grade aradora, foi usada uma grade de 20 discos, incorporando-se ao solo os resíduos até $15 \mathrm{~cm}$. O plantio direto foi feito com plantadora apropriada, a qual proporcionou a abertura de pequenos sulcos para a deposição das sementes, deixando todo o resíduo das culturas na superfície do solo. No início do quarto ano de plantio foram aplicadas $2,5 \mathrm{tha}^{-1}$ de calcário em toda área experimental. Nos tratamentos de arado e de grade, o corretivo foi incorporado ao solo, e no plantio direto permaneceu na superfície. Aplicaram-se $400 \mathrm{~kg} \mathrm{ha}^{-1}$, da fórmula 5-30-5 por ocasião da semeadura de cada cultura.

Uma amostra para análise química foi coletada no final de 1996, nos três tratamentos, em uma malha quadrada de 49 pontos $(7 \times 7)$, a espaços de $4 \times 4 \mathrm{~m}$, ou seja, uma amostra por ponto, nas profundidades de $0-5 \mathrm{~cm}$ e $5-20 \mathrm{~cm}$, sendo estas profundidades definidas no sentido de verificar, ou não, o acúmulo superficial de fertilizantes. Foram determinados $\mathrm{pH}, \mathrm{P}, \mathrm{K}, \mathrm{Ca}$, e $\mathrm{Mg}$, conforme Embrapa (1997). Os dados foram analisados, calculando-se média, coeficiente de variação, valores mínimo e máximo, assimetria e curtose, nas duas profundidades e nos três sistemas de preparo.

A Estatística clássica assume que a variação das características do solo dentro das unidades amostrais não são correlacionadas, e que a média das amostras é o melhor estimador das características de solo em qualquer local na unidade amostral. O número de amostras necessário para estimar o valor médio das características de solo, segundo Cochran (1965), é definido por:

$n=t^{2}{ }_{\alpha} s^{2} / d^{2}$

em que $n$ é o número de amostras necessário para estimar a média $\mu$, dentro de uma tolerância d. A quantidade $t_{\alpha}$ é a estatística t de Student, referente a um determinado nível de probabilidade $\alpha$, e s ${ }^{2}$ é a variância amostral. Normalmente, o uso da Estatística clássica requer maior número de amostras do que a Geoestatística, para estimar o parâmetro em estudo com determinada precisão. Por isso, McBratney \& Webster (1983), Vieira et al. (1983) e Souza et al. (1997) preconizam que o estudo da dependência espacial das propriedades do solo pode reduzir o número de amostras em relação ao uso dos procedimentos amostrais definidos na Estatística clássica. Com esta preocupação em mente, a amostragem casual simples poderia, por exemplo, ser evitada, visto que existe a probabilidade de coletar amostras em locais similares, duplicando dessa forma a informação. A dependência espacial entre as observações pode ser expressa através do semivariograma, estimado pela seguinte equação: 
$\gamma^{*}(\mathrm{~h})=\frac{1}{2 \mathrm{~N}(\mathrm{~h})} \sum_{\mathrm{i}=1}^{\mathrm{N}(\mathrm{h})}\left[\mathrm{Z}\left(\mathrm{x}_{\mathrm{i}}\right)-\mathrm{Z}\left(\mathrm{x}_{\mathrm{i}}+\mathrm{h}\right)\right]^{2}$,

em que $\mathrm{N}(\mathrm{h})$ é o número de pares de valores medidos $\mathrm{Z}\left(\mathrm{x}_{\mathrm{i}}\right)$, $\mathrm{Z}\left(\mathrm{x}_{\mathrm{i}}+\mathrm{h}\right)$, separados pela distância $\mathrm{h}$, se a variável for escalar (Vieira et al., 1983). O gráfico de $\gamma^{*}(\mathrm{~h})$ versus os valores correspondentes de h, chamado semivariograma, é uma função do vetor $\mathrm{h}$, e portanto depende de ambos, magnitude e direção de h. Modelos matemáticos devem ser ajustados aos semivariogramas, os quais permitem visualizar a natureza da variação espacial das variáveis estudadas, além de serem necessários para outras aplicações, como, por exemplo, krigagem.

Os seguintes modelos matemáticos foram ajustados aos semivariogramas:

a) Modelo esférico:

$\gamma(\mathrm{h})=\mathrm{C}_{0}+\mathrm{C}_{1}\left[\frac{3}{2}\left(\frac{\mathrm{h}}{\mathrm{a}}\right)-\frac{1}{2}\left(\frac{\mathrm{h}}{\mathrm{a}}\right)^{3}\right], \quad 0<\mathrm{h}<\mathrm{a}$

$\gamma(\mathrm{h})=\mathrm{C}_{\mathrm{o}}+\mathrm{C}_{1}, \mathrm{~h} \geq \mathrm{a}$;

b) Modelo exponencial:

$$
\gamma(\mathrm{h})=\mathrm{C}_{0}+\mathrm{C}_{1}\left[1-\exp \left(-3 \frac{\mathrm{h}}{\mathrm{a}}\right)\right], \quad 0<\mathrm{h}<\mathrm{d}
$$

em que dé a máxima distância na qual o semivariograma é definido;

c) Modelo gaussiano:

$$
\gamma(\mathrm{h})=\mathrm{C}_{0}+\mathrm{C}_{1}\left[1-\exp \left(-3\left(\frac{\mathrm{h}}{\mathrm{a}}\right)^{2}\right)\right], 0<\mathrm{h}<\mathrm{d} \text {. }
$$

Nos três modelos acima, $\mathrm{C}_{0}$ é o efeito pepita, $\mathrm{C}_{0}+\mathrm{C}_{1}$ é o patamar, e a é o alcance do semivariograma.

Ajustar modelos matemáticos aos semivariogramas é um procedimento subjetivo. A qualidade de ajuste pode ser verificada através da técnica de "jack-knifing". Como para cada local têm-se um valor medido e pode-se estimar outro valor por meio da krigagem ou co-krigagem, pode-se calcular a regressão linear entre estes pares de dados e calcular a interseção (a), o coeficiente angular (b), a correlação entre os pares $\left(\mathrm{r}^{2}\right)$, o erro absoluto com sua média e variância, e o erro reduzido com sua média e variância (Vieira et al., 1983; Vieira, 1997). O melhor ajuste se obtém quando os valores obtidos se aproximam dos seguintes valores ideais: $\mathrm{a}=0 ; \mathrm{b}=1 ; \mathrm{r}^{2}=1$; média do erro absoluto $=0$; variância do erro absoluto $=$ mínima; média do erro reduzido $=0 ;$ e variância do erro reduzido $=1$.

Os valores obtidos através da krigagem não são viciados e têm variância mínima (Vieira et al., 1983) e ideais para a construção de mapas de isolinhas ou tridimensionais para verificação e interpretação da variabilidade espacial. As informações mostradas nestes gráficos de isolinhas são muito úteis para melhor entender a variabilidade das pro- priedades do solo no campo e para identificar áreas que necessitam diferentes tipos de preparo.

\section{Resultados e Discussão}

Valores teóricos de assimetria e curtose de 0 e 3, respectivamente, indicam a normalidade dos dados (Tabela 1). A estimação por krigagem apresenta melhores resultados quando a normalidade dos dados é satisfeita (Paz-Gonzalez et al., 2001). Quarenta porcento das 30 combinações possíveis (cinco variáveis, duas profundidades de coleta e três sistemas de preparo) apresentam valores de assimetria e curtose não compatíveis com a distribuição normal. Quanto às demais combinações, todos têm assimetria e curtose próximos o suficiente de 0 e 3 para serem considerados como distribuições normais. A combinação da profundidade 5-20 cm com o sistema plantio direto é onde se têm mais variáveis com distribuição não-normal. Somente para $\mathrm{pH}$ nas diversas combinações, o coeficiente de variação manteve-se baixo $(<10 \%)$, classificação sugerida por Pimentel-Gomes (1984) (Tabela 1). As demais variáveis, em todas as outras combinações, apresentaram coeficientes de variação altos $(>20 \%)$, com exceção de Ca quanto à profundidade $0-5 \mathrm{~cm}$ nos tratamentos grade aradora e plantio direto, que apresentaram coeficiente de variação médio (entre 10\% e 20\%). Uma possível explicação para esta variabilidade seria o fato de se ter feito uma amostragem simples nos 49 pontos amostrados. Com amostragem composta, provavelmente, seriam encontradas menores variabilidades. Entretanto, a variação na pequena distância pode ser uma informação importante principalmente para os elementos que têm mobilidade pequena.

Foi encontrada correlação espacial em 16 das 30 combinações possíveis de tratamentos, profundidades e nutriente (Tabela 2). As demais combinações de variáveis nas diferentes profundidades e diferentes sistemas de preparo apresentaram efeito pepita puro, ou seja, ausência de dependência espacial, indicando distribuição espacial aleatória. Nestes casos, a Estatística clássica pode ser aplicada (Silva et al., 1989). Os modelos esférico, exponencial e gaussiano, respectivamente equações 3,4 e 5, ajustados aos semivariogramas, foram submetidos ao processo de validação “jack-knifing”, e selecionados os mais compatíveis com os indicadores ideais. Das 16 combinações entre variáveis, profundidades 
e sistemas de preparo, com correlação espacial, sete apresentaram isotropia, ou seja, variabilidade independente da direção escolhida na parcela experimental (Tabela 2). Entretanto, devido à falta de estacionaridade para a combinação da variável $\mathrm{P}$, para arado e profundidades de 0-5 e 5-20 cm, foram calculados os semivariogramas nos resíduos da tendência. Após a remoção da tendência, o semivariograma relativo ao $P$ na profundidade $0-5 \mathrm{~cm}$ apresentou um efeito pepita maior $(0,41)$, com um alcance também maior (11). O modelo esférico ajustado às semivariâncias escalonadas predominou em cinco das sete combinações (Tabela 2). Nas duas restantes, o modelo exponencial foi o que melhor se ajustou. Quanto às demais combinações (9), foram ajustados semivariogramas direcionais não-escalonados, e a dependência espacial foi melhor representada na direção de Y (Tabela 2). O semivariograma onidirecional

Tabela 1. Valores mínimos, máximos e médios de $\mathrm{pH}\left(\mathrm{H}_{2} \mathrm{O}\right), \mathrm{Ca}^{2+}\left(\mathrm{cmol}_{\mathrm{c}} \mathrm{dm}^{-3}\right), \mathrm{Mg}^{2+}\left(\mathrm{cmol}_{\mathrm{c}} \mathrm{dm}^{-3}\right), \mathrm{P}^{\left(\mathrm{mg} \mathrm{dm}^{-3}\right)} \mathrm{e}$ $\mathrm{K}^{+}\left(\mathrm{mg} \mathrm{dm}^{-3}\right)$ em razão dos tratamentos de preparo do solo, nas profundidades de 0-5 e 5-20 cm, e valores do coeficiente de variação $(\mathrm{CV})$, assimetria e curtose dos dados.

\begin{tabular}{|c|c|c|c|c|c|c|}
\hline Variável $^{(1)}$ & Mínimo & Máximo & Média & CV (\%) & Assimetria & Curtose \\
\hline \multicolumn{7}{|c|}{$0-5 \mathrm{~cm}-$ arado } \\
\hline pH & 5,30 & 8,30 & 5,87 & 3,83 & $-0,14$ & 1,65 \\
\hline $\mathrm{Ca}[\mathrm{N}]$ & 1,00 & 2,50 & 1,76 & 20,22 & 0,11 & 2,59 \\
\hline $\operatorname{Mg}[\mathrm{N}]$ & 0,40 & 1,60 & 0,92 & 25,36 & 0,49 & 3,67 \\
\hline $\mathrm{P}[\mathrm{N}]$ & 1,00 & 25,80 & 9,05 & 59,17 & 0,89 & 3,81 \\
\hline $\mathrm{K}$ & 42,00 & 194,00 & 78,96 & 35,93 & 2,04 & 8,44 \\
\hline \multicolumn{7}{|c|}{ Grade aradora } \\
\hline $\mathrm{pH}$ & 5,40 & 6,70 & 5,79 & 4,08 & 0,90 & 6,25 \\
\hline $\mathrm{Ca}[\mathrm{N}]$ & 1,10 & 2,20 & 1,71 & 17,09 & $-0,29$ & 2,01 \\
\hline $\mathrm{Mg}$ & 0,40 & 2,00 & 0,96 & 28,55 & 1,17 & 5,48 \\
\hline $\mathrm{P}[\mathrm{N}]$ & 10,30 & 34,80 & 21,91 & 21,83 & $-0,48$ & 3,73 \\
\hline $\mathrm{K}[\mathrm{N}]$ & 25,00 & 189,00 & 104,60 & 40,17 & 0,25 & 2,09 \\
\hline \multicolumn{7}{|c|}{ Plantio direto } \\
\hline $\mathrm{pH}[\mathrm{N}]$ & 5,40 & 6,90 & 6,37 & 5,34 & $-0,62$ & 2,89 \\
\hline $\mathrm{Ca}[\mathrm{N}]$ & 1,60 & 4,10 & 3,17 & 18,73 & $-0,31$ & 2,72 \\
\hline $\mathrm{Mg}[\mathrm{N}]$ & 0,60 & 1,90 & 1,31 & 21,36 & $-0,036$ & 2,64 \\
\hline $\mathrm{P}[\mathrm{N}]$ & 5,80 & 112,80 & 39,80 & 62,58 & 0,84 & 3,28 \\
\hline $\mathrm{K}$ & 65,00 & 292,00 & 146,70 & 28,25 & 0,93 & 4,71 \\
\hline \multicolumn{7}{|c|}{$5-20 \mathrm{~cm}$ - arado } \\
\hline $\mathrm{pH}[\mathrm{N}]$ & 5,40 & 6,30 & 5,87 & 3,72 & 0,12 & 2,43 \\
\hline $\mathrm{Ca}[\mathrm{N}]$ & 1,00 & 2,40 & 1,74 & 20,07 & $-0,26$ & 2,50 \\
\hline $\operatorname{Mg}[\mathrm{N}]$ & 0,40 & 1,40 & 0,90 & 26,09 & 0,15 & 2,34 \\
\hline $\mathrm{P}$ & 0,50 & 36,10 & 9,32 & 69,41 & 1,71 & 7,33 \\
\hline K & 47,00 & 223,00 & 86,37 & 37,96 & 1,90 & 7,90 \\
\hline \multicolumn{7}{|c|}{ Grade aradora } \\
\hline $\mathrm{pH}[\mathrm{N}]$ & 4,90 & 6,00 & 5,59 & 4,36 & $-0,37$ & 2,78 \\
\hline $\mathrm{Ca}[\mathrm{N}]$ & 0,80 & 2,40 & 1,56 & 21,07 & 0,11 & 2,69 \\
\hline $\operatorname{Mg}[N]$ & 0,30 & 1,30 & 0,80 & 29,01 & 0,039 & 2,51 \\
\hline $\mathrm{P}$ & 4,90 & 75,20 & 14,98 & 73,98 & 3,52 & 18,51 \\
\hline $\mathrm{K}[\mathrm{N}]$ & 20,00 & 172,00 & 98,22 & 32,92 & $-0,062$ & 3,12 \\
\hline \multicolumn{7}{|c|}{ Plantio direto } \\
\hline $\mathrm{pH}[\mathrm{N}]$ & 4,70 & 5,90 & 5,40 & 5,34 & $-0,50$ & 2,75 \\
\hline $\mathrm{Ca}$ & 0,40 & 2,60 & 1,38 & 28,64 & 0,50 & 4,09 \\
\hline $\mathrm{Mg}$ & 0,10 & 1,40 & 0,64 & 37,10 & 0,68 & 4,34 \\
\hline $\mathrm{P}$ & 2,40 & 131,50 & 19,58 & 135,50 & 2,44 & 8,81 \\
\hline $\mathrm{K}$ & 25,00 & 137,00 & 52,49 & 50,19 & 1,64 & 5,29 \\
\hline
\end{tabular}

${ }^{(1)} \mathrm{N}$ : identifica variável normalmente distribuída. 
apresentou efeito pepita puro. Entretanto, o método de "jack-knifing" garantiu o uso dos semivariogramas na direção de Y. A razão de tal comportamento se deve, provavelmente, a práticas culturais, com as operações de campo realizadas nesta direção.

Das combinações que apresentaram dependência espacial, somente quatro (Tabela 2) podem ser consideradas como forte dependência (efeito pepita $\leq 25 \%$ do patamar), e o restante, como moderada (efeito pepita entre $26-75 \%$ do patamar), segundo Cambardella et al. (1994). Os semivariogramas relativos às combinações $(\mathrm{pH}$, arado, $5-20 \mathrm{~cm} ; \mathrm{Mg}$, grade, $0-5 \mathrm{~cm}$; $\mathrm{P}$, grade, $0-5 \mathrm{~cm}$ e $\mathrm{pH}$, grade, $5-20 \mathrm{~cm}$ ) com forte dependência espacial estão representados na Figura 1. Constata-se uma associação entre o uso do arado como sistema de preparo e a direção de anisotropia $\left(90^{\circ}\right)$, sendo que a profundidade $5-20 \mathrm{~cm}$ apresentou a maior dependência espacial (7\%) (Tabela 2). O semivariograma para $\mathrm{K} \mathrm{a} 0-5 \mathrm{~cm}$, sob preparo com arado, apresentou maior alcance. Entretanto, quando os semivariogramas isotrópicos são considerados, o sistema de preparo que apresenta as maiores dependências espaciais é a grade (16\% para
$\mathrm{Mg}$ e $\mathrm{P}$ na profundidade $0-5 \mathrm{~cm}$ e $24 \%$ para $\mathrm{pH}$ na profundidade 5-20 $\mathrm{cm}$ ) (Tabela 2).

Existe grande variação quanto à dependência espacial das propriedades químicas no solo conforme o método de preparo e profundidade. Apenas as variáveis $\mathrm{P}$ e $\mathrm{K}$ apresentam dependência espacial na profundidade de $0-5 \mathrm{~cm}$, independentemente do sistema de preparo do solo (Tabela 2). São as que apresentam maior coeficiente de variação, demonstrando sua grande variabilidade em torno da média (Tabela 1). Entretanto, o mesmo resultado não se aplica para a profundidade $5-20 \mathrm{~cm}$, o que indica que a profundidade é um fator importante no estudo da dependência espacial. No caso do $\mathrm{P}$, a baixa mobilidade no perfil condiciona estes resultados, independentemente do preparo. Quanto ao K, apesar de a variabilidade ser bastante dependente do preparo em virtude da sua mobilidade, tal conclusão não foi observada. Segundo Salet et al. (1997) “a alta variabilidade do P no solo está relacionada com as linhas de adubação. A variabilidade do K no solo não deve estar relacionada somente às linhas de adubação, mas também, à localização das plantas, pois esse ele-

Tabela 2. Estimativas dos parâmetros dos modelos dos semivariogramas ajustados para as variáveis $\mathrm{Ca}^{2+}\left(\mathrm{cmol}_{\mathrm{c}} \mathrm{dm}^{-3}\right)$, $\mathrm{K}^{+}\left(\mathrm{mg} \mathrm{dm}^{-3}\right), \mathrm{pH}\left(\mathrm{H}_{2} \mathrm{O}\right), \mathrm{Mg}^{2+}\left(\mathrm{cmol}_{\mathrm{c}} \mathrm{dm}^{-3}\right)$ e $\mathrm{P}\left(\mathrm{mg} \mathrm{dm}^{-3}\right)$ em diferentes sistemas de preparo nas profundidades 0-5 e $5-20 \mathrm{~cm}$.

\begin{tabular}{|c|c|c|c|c|c|c|}
\hline Sistema & $\begin{array}{l}\text { Profundidade } \\
(\mathrm{cm})\end{array}$ & $\mathrm{C}_{0}$ & $\mathrm{C}_{1}$ & $\mathrm{a}$ & Modelo $^{(1)}$ & $\begin{array}{c}\text { Dependência } \\
{\left[\mathrm{C}_{0} /\left(\mathrm{C}_{0}+\mathrm{C}_{1}\right)\right] \times 100}\end{array}$ \\
\hline \multicolumn{7}{|c|}{$\mathrm{Ca}^{2+}$} \\
\hline Arado & $0-5$ & 0,011 & 0,028 & 14 & Esférico[D] & 28 \\
\hline Plantio direto & $0-5$ & 0,15 & 0,15 & 15 & Esférico[D] & 50 \\
\hline Arado & $5-20$ & 0,03 & 0,03 & 14 & Gauss[D] & 50 \\
\hline Grade & $5-20$ & 0,03 & 0,045 & 10 & Exponencial[D] & 40 \\
\hline \multicolumn{7}{|c|}{$\mathrm{K}^{+}$} \\
\hline Arado & $0-5$ & 280 & 580 & 17 & Exponencial[D] & 33 \\
\hline Grade & $0-5$ & 700 & 700 & 14 & Gauss[D] & 50 \\
\hline Plantio direto & $0-5$ & 600 & 400 & 14 & Exponencial[D] & 60 \\
\hline \multicolumn{7}{|c|}{$\mathrm{pH}\left(\mathrm{H}_{2} \mathrm{O}\right)$} \\
\hline Arado & $0-5$ & 0,011 & 0,014 & 9 & Exponencial[D] & 44 \\
\hline Arado & $5-20$ & 0,01 & 0,15 & 14 & Exponencial[D] & 7 \\
\hline Grade & $5-20$ & 0,25 & 0,77 & 11 & Exponencial & 24 \\
\hline \multicolumn{7}{|c|}{$\mathrm{Mg}^{2+}$} \\
\hline Grade & $0-5$ & 0,15 & 0,8 & 6,5 & Esférico & 16 \\
\hline Grade & $5-20$ & 0,39 & 0,6 & 11 & Esférico & 39 \\
\hline \multicolumn{7}{|c|}{$\mathrm{P}$} \\
\hline Arado & $0-5$ & 0,41 & 0,65 & 11 & Esférico[R] & 39 \\
\hline Arado & $5-20$ & 0,39 & 0,65 & 7 & Esférico[R] & 38 \\
\hline Grade & $0-5$ & 0,15 & 0,8 & 6,5 & Esférico & 16 \\
\hline Plantio direto & $0-5$ & 0,33 & 0,75 & 9 & Exponencial & 31 \\
\hline
\end{tabular}

(1)D: semivariogramas anisotrópicos na direção de Y; R: variável com retirada de tendência. 
mento não forma compostos orgânicos no tecido das plantas, e é facilmente transportado da parte aérea para o solo, após a chuva. Desse modo, tende a concentrar maiores teores próximo ao colo da planta, e diminui com o afastamento da mesma". Não existe na profundidade de $5-20 \mathrm{~cm}$, qualquer propriedade química do solo, que apresente dependência espacial em relação aos três sistemas de preparo.

O alcance é de fundamental importância para a interpretação dos semivariogramas. Ele indica a distância até onde os pontos amostrais estão correlacionados entre si (Vieira et al., 1983; Souza et al., 1997; Vieira, 1997), ou seja, os pontos localizados em uma área cujo raio seja o alcance, são mais semelhantes entre si, do que os separados por distâncias maiores.

Segundo McBratney \& Webster (1983) e Souza et al. (1997), uma das principais utilizações dos semivariogramas é na determinação do número ideal de amostras para a estimação das características químicas do solo. Com o conhecimento do alcance da dependência espacial, define-se o raio de amostragem, e, para garantir em uma amostragem futura nas mesmas condições do experimento em questão, a independência dos pontos amostrais, a minimização do erro-padrão da média e também para diminuir o trabalho na coleta e no número de amostras, o intervalo de amostragem deve ser duas vezes o alcance. Entretanto, a independência dos pontos amostrais garantiriam a construção do histograma, mas não trariam informações sobre a distribuição espacial dos valores a eles alocado.

O plano de amostragem poderia incluir "cercas de pontos" em diversas direções, fornecendo maior precisão no valor do efeito pepita, devido à proximidade das amostras, mas apresentaria o inconveniente de estar sendo assumido que estas "cercas de pontos" apresentariam os mesmos resultados em qualquer posição dentro da parcela, o que, não se pode comprovar por este trabalho.

Para um futuro experimento na mesma área e nas mesmas condições, os resultados obtidos pelo uso da Geoestatística (Tabela 2) e Estatística clássica (Tabela 3) podem servir de subsídio para a determinação do número de amostras (equação 1) nos diversos níveis de probabilidade. Se fixar por exemplo, a variável $\mathrm{P}$, com um sistema de preparo com arado a uma profundidade de $5-20 \mathrm{~cm}$, verifica-se que o al-
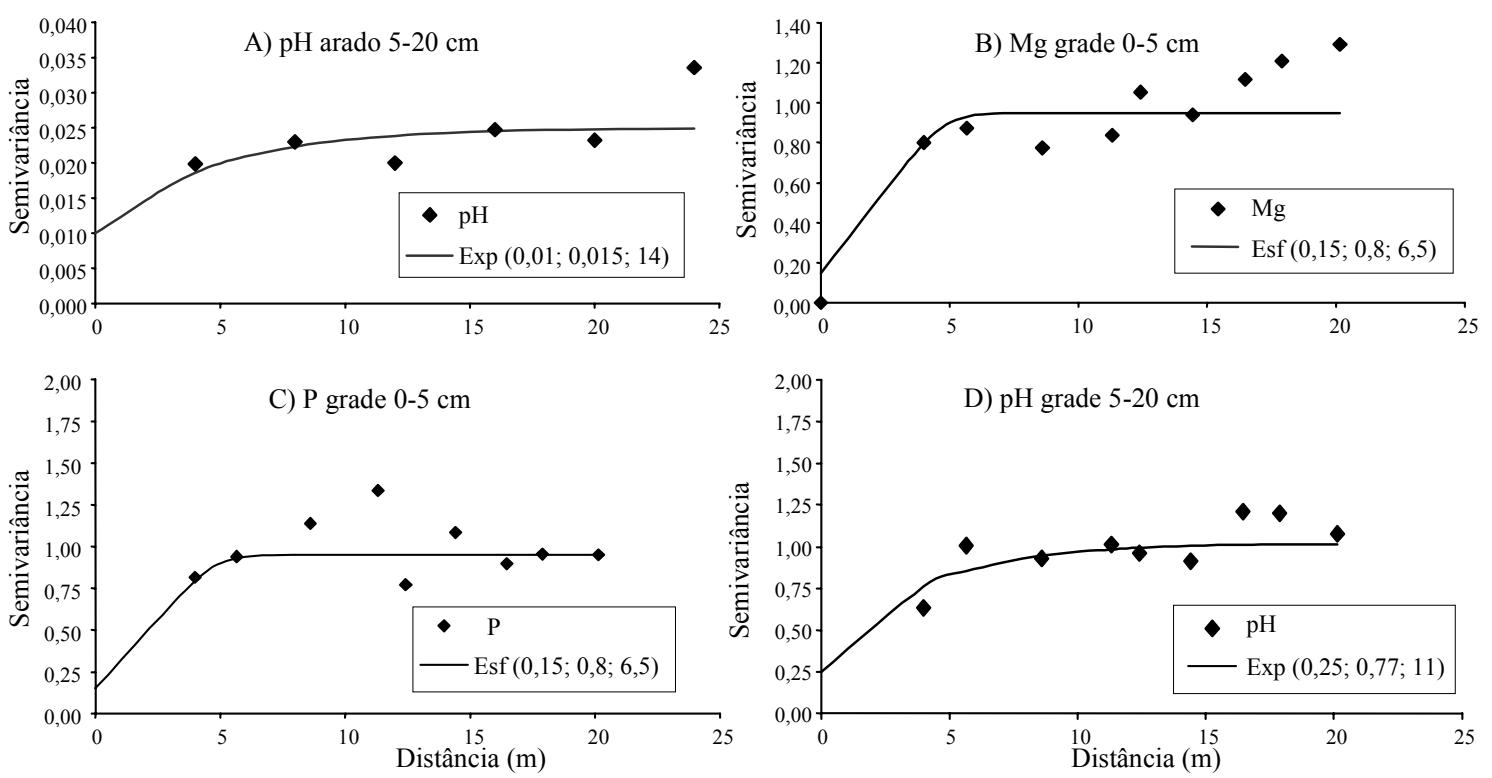

Figura 1. Semivariogramas para $\mathrm{pH}, \mathrm{Mg}$ e $\mathrm{P}$ em diferentes sistemas de preparo e diferentes profundidades em Latossolo Vermelho-Escuro distrófico: A) modelo exponencial (Exp) ajustado na direção de Y para pH; B) modelo esférico (Esf) ajustado para $\mathrm{Mg}$; C) modelo esférico ajustado para $\mathrm{P}$; D) modelo exponencial ajustado para $\mathrm{pH}$. 
cance é de 7 m (Tabela 2). Para garantir a dependência espacial, pontos de amostragem deveriam ser coletados a uma distância equivalente à metade do alcance, de 3,5 a $4 \mathrm{~m}$, o que foi realizado no plano amostral delineado. O alcance do semivariograma garante a construção de mapas com 49 amostras, número bem menor do que as 787 amostras obtidas pela Estatística clássica (Tabela 3).
Os gráficos de isolinhas estimados por krigagem (Vieira et al., 1983) das variáveis com maior dependência espacial são apresentados na Figura 2, com os valores agrupados em cinco classes em ordem crescente.

Somente pelo conhecimento da variabilidade no campo experimental é que adequados esquemas de amostragem podem ser definidos. Estas figuras mos-

Tabela 3. Número de subamostras para estimar os valores de $\mathrm{pH}\left(\mathrm{H}_{2} \mathrm{O}\right), \mathrm{Ca}^{2+}\left(\mathrm{cmol}_{\mathrm{c}} \mathrm{dm}^{-3}\right), \mathrm{Mg}^{2+}\left(\mathrm{cmol}_{\mathrm{c}} \mathrm{dm}^{-3}\right)$, $\mathrm{P}\left(\mathrm{mg} \mathrm{dm}^{-3}\right)$ e $\mathrm{K}^{+}\left(\mathrm{mg} \mathrm{dm}^{-3}\right)$, para os diferentes sistemas de preparo do solo (arado, grade aradora e plantio direto) nas profundidades $0-5$ e $5-20 \mathrm{~cm}^{(1)}$.

\begin{tabular}{|c|c|c|c|c|c|c|}
\hline \multirow[t]{2}{*}{ Variável } & \multicolumn{6}{|c|}{ Número de subamostras referente à variação em torno da média } \\
\hline & $5 \%$ & $10 \%$ & $15 \%$ & $20 \%$ & $25 \%$ & $30 \%$ \\
\hline \multicolumn{7}{|c|}{$0-5 \mathrm{~cm}-$ arado } \\
\hline $\mathrm{pH}$ & 3 & 1 & 1 & 1 & 1 & 1 \\
\hline $\mathrm{Ca}$ & 67 & 17 & 8 & 5 & 3 & 2 \\
\hline $\mathrm{Mg}$ & 106 & 27 & 12 & 7 & 5 & 3 \\
\hline $\mathrm{P}$ & 572 & 143 & 64 & 36 & 23 & 16 \\
\hline $\mathrm{K}$ & 211 & 53 & 24 & 14 & 9 & 6 \\
\hline \multicolumn{7}{|c|}{ Grade aradora } \\
\hline $\mathrm{pH}$ & 3 & 1 & 1 & 1 & 1 & 1 \\
\hline $\mathrm{Ca}$ & 48 & 12 & 6 & 3 & 2 & 2 \\
\hline $\mathrm{Mg}$ & 134 & 34 & 15 & 9 & 6 & 4 \\
\hline$P$ & 79 & 20 & 9 & 5 & 4 & 3 \\
\hline K & 264 & 66 & 30 & 17 & 11 & 8 \\
\hline \multicolumn{7}{|c|}{ Plantio direto } \\
\hline $\mathrm{pH}$ & 5 & 2 & 1 & 1 & 1 & 1 \\
\hline $\mathrm{Ca}$ & 58 & 15 & 7 & 4 & 3 & 2 \\
\hline $\mathrm{Mg}$ & 75 & 19 & 9 & 5 & 3 & 3 \\
\hline $\mathrm{P}$ & 640 & 160 & 72 & 40 & 26 & 18 \\
\hline $\mathrm{K}$ & 131 & 33 & 15 & 9 & 6 & 4 \\
\hline \multicolumn{7}{|c|}{$5-20 \mathrm{~cm}$ - arado } \\
\hline $\mathrm{pH}$ & 3 & 1 & 1 & 1 & 1 & 1 \\
\hline $\mathrm{Ca}$ & 66 & 17 & 8 & 5 & 3 & 2 \\
\hline $\mathrm{Mg}$ & 112 & 28 & 13 & 7 & 5 & 4 \\
\hline $\mathrm{P}$ & 787 & 197 & 88 & 50 & 32 & 22 \\
\hline K & 236 & 59 & 27 & 15 & 10 & 7 \\
\hline \multicolumn{7}{|c|}{ Grade aradora } \\
\hline $\mathrm{pH}$ & 4 & 1 & 1 & 1 & 1 & 1 \\
\hline $\mathrm{Ca}$ & 73 & 19 & 9 & 5 & 3 & 3 \\
\hline $\mathrm{Mg}$ & 138 & 35 & 16 & 9 & 6 & 4 \\
\hline $\mathrm{P}$ & 894 & 224 & 100 & 56 & 36 & 25 \\
\hline $\mathrm{K}$ & 178 & 45 & 20 & 12 & 8 & 5 \\
\hline \multicolumn{7}{|c|}{ Plantio direto } \\
\hline $\mathrm{pH}$ & 5 & 2 & 1 & 1 & 1 & 1 \\
\hline $\mathrm{Ca}$ & 134 & 34 & 15 & 9 & 6 & 4 \\
\hline $\mathrm{Mg}$ & 225 & 57 & 25 & 15 & 9 & 7 \\
\hline $\mathrm{P}$ & 2.998 & 750 & 334 & 188 & 120 & 84 \\
\hline K & 412 & 103 & 46 & 26 & 17 & 12 \\
\hline
\end{tabular}

${ }^{(1)}$ Tabela adaptada de Silveira et al. (2000), para o nível de probabilidade de $95 \%$. 
tram que a variabilidade se acentua na direção $X$, na direção da declividade do solo, e que a amostragem ao acaso possivelmente não representaria todas as variações encontradas; daí a importância da amostragem sistemática em uma malha regular. Tam-
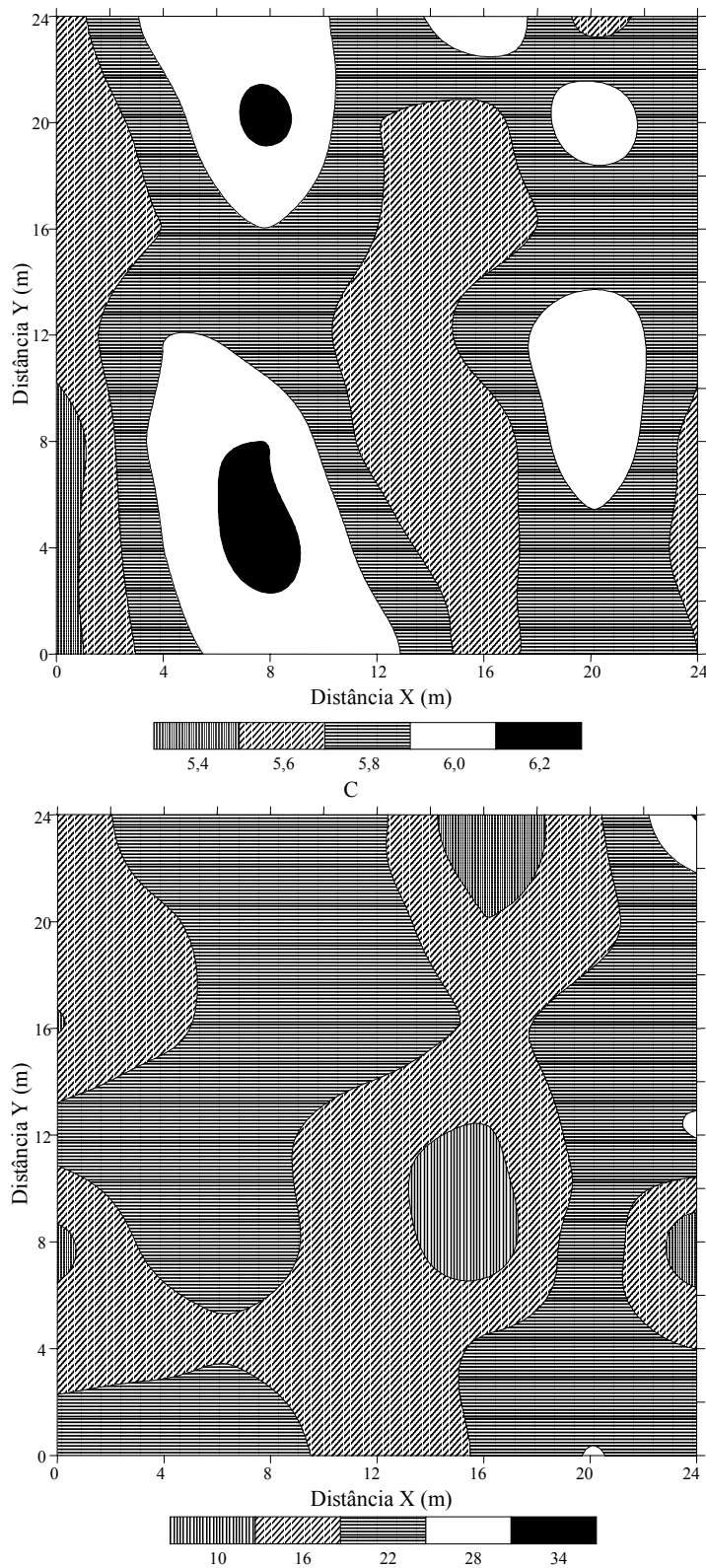

bém fica claro que existe uma grande variabilidade dentro da parcela, e portanto, o valor médio pode não ser a melhor representação da população, contrariando uma das pressuposições da estatística clássica, ou seja, que as parcelas devem ser as mais
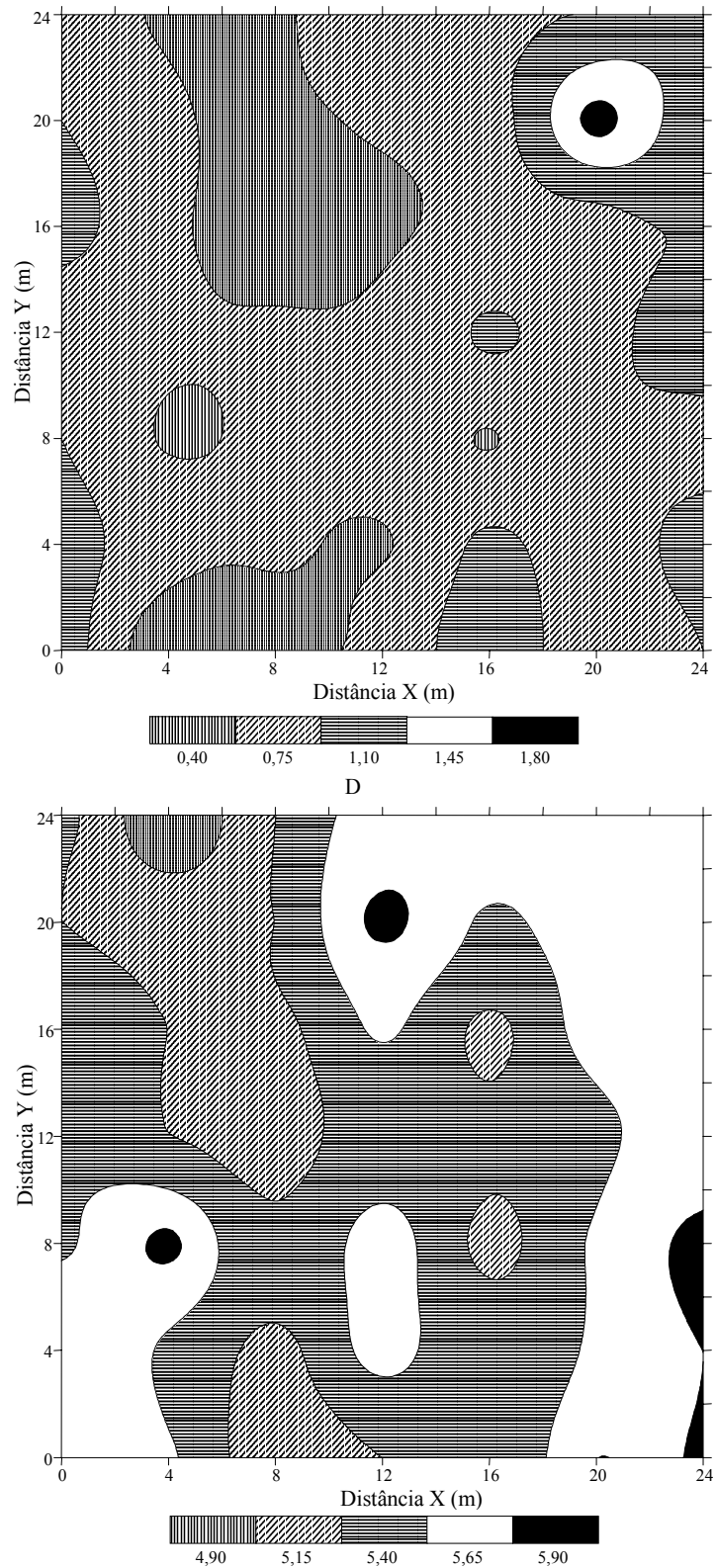

Figura 2. Mapa de isolinhas de $\mathrm{pH}, \mathrm{Mg}, \mathrm{P}$, relativas a diferentes sistemas de preparo e profundidades em Latossolo Vermelho-Escuro distrófico: A) $\mathrm{pH}$, preparado com arado a 5-20 $\mathrm{cm}$ de profundidade; B) $\mathrm{Mg}$, preparado com grade a 0-5 $\mathrm{cm}$ de profundidade; C) P, preparado com grade a 0-5 $\mathrm{cm}$ de profundidade; $\mathrm{D}$ ) $\mathrm{pH}$, preparado com grade a $5-20 \mathrm{~cm}$ de profundidade. 
homogêneas possível, para que a média possa ser a estatística usada na representação dos tratamentos alocados à parcela. Vieira (1997) examinou a variabilidade espacial de alguns atributos químicos e granulométricos do solo em uma parcela de $30 \times 30 \mathrm{~m}$, e concluiu, devido à variabilidade encontrada, que a amostragem ao acaso não conseguiria detectá-la.

\section{Conclusões}

1. Somente o $\mathrm{pH}$ apresenta baixa variabilidade nos três sistemas de preparo e nas duas profundidades estudadas.

2. A dependência espacial é melhor representada na direção de $\mathrm{Y}$, devido a práticas culturais, com as operações de campo realizadas nesta direção.

3. A direção de anisotropia está associada com o uso de arado e com a topografia do terreno, porém as mais fortes dependências espaciais, quando a isotropia é considerada, ocorrem quando o sistema de preparo é a grade.

4. As variáveis $\mathrm{P}$ e $\mathrm{K}$ apresentam dependência espacial para $0-5 \mathrm{~cm}$ de profundidade de solo, independentemente do sistema de preparo; tais resultados não se repetem na profundidade $5-20 \mathrm{~cm}$, o que indica que a dependência espacial varia conforme a profundidade de coleta da amostra do solo.

\section{Referências}

BURROUGH, P. A.; BOUMA, J.; YATES, S. R. The state of the art in pedometrics. Geoderma, Amsterdam, v. 62, n. 1/3, p. 311-326, 1994.

CAMBARDELLA, C. A.; MOORMAN, T. B.; NOVAK, J. M.; PARKIN, T. B.; KARLEN, D. L.; TURCO, R. F.; KONOPKA, A. E. Field-scale variability of soil properties in central Iowa soils. Soil Science Society of America Journal, Madison, v. 58, n. 5, p. 1501-1511, 1994.

CARVALHO, J. R. P. de. Amostragem de solo em plantio direto para aplicação em agricultura de precisão. Campinas: Embrapa-CNPTIA, 1999. 4 p. (Pesquisa em Andamento, 1).

CHUNG, C. K.; CHONG, S. K.; VARSA, E. C. Sampling strategies for fertility on a stoy silt loam soil. Communications in Soil Science and Plant Analysis, New York, v. 26. n. 5/6, p. 741-763, 1995.

COCHRAN, W. G. Téenicas de amostragem. Rio de Janeiro: Fundo de Cultura, 1965. 555 p.
EMBRAPA. Centro Nacional de Pesquisa de Solos (Rio de Janeiro, RJ). Manual de métodos de análise de solo. 2. ed. rev. atual. Rio de Janeiro, 1997. 212 p. (Documentos, 1).

McBRATNEY, A. B.; WEBSTER, R. How many observations are needed for regional estimation of soil properties? Soil Science, Baltimore, v. 135, n. 3, p. 177183, 1983.

ORLANDO FILHO, J.; RODELLA, A. A análise química do solo e recomendação de adubação. In: ORLANDO FILHO, J. (Coord.). Nutrição e adubação da cana-deaçúcar no Brasil. Piracicaba: Instituto do Açúcar e do Álcool/Planalsucar, 1983. p. 155-178.

PAZ-GONZALEZ, A.; TABOADA CASTRO, M. T.; VIEIRA, S. R. Geostatistical analysis of heavy metals in a one-hectare plot under natural vegetation in a serpentine area. Canadian Journal of Soil Science, Ottawa, v. 81, p. 469-479, 2001.

PIMENTEL-GOMES, F. A estatística moderna na pesquisa agropecuária. Piracicaba: Associação Brasileira para Pesquisa da Potassa e do Fosfato, 1984. 160 p.

SALET, R. L.; ANGHINONI, I.; KOCHHANN, R. A.; KRAY, C. H.; CONTE, E. Como fazer uma amostragem de solo no sistema plantio direto. In: SEMINÁRIO INTERNACIONAL DO SISTEMA PLANTIO DIRETO, 2., 1997, Passo Fundo. Anais... Passo Fundo: EmbrapaCNPT, 1997. p. 205-207.

SILVA, A. P. da; LIBARDI, P. L.; VIEIRA, S. R. Variabilidade espacial da resistência à penetração de um Latossolo Vermelho-Escuro ao longo de uma transeção. Revista Brasileira de Ciência do Solo, Campinas, v. 13, n. 1, p. 1-5, 1989.

SILVEIRA, P. M. da; ZIMMERMANN, F. J. P.; SILVA, S. C. da; CUNHA, A. A. da. Amostragem e variabilidade espacial de características químicas de um latossolo submetido a diferentes sistemas de preparo. Pesquisa Agropecuária Brasileira, Brasília, v. 35, n. 10, p. 2057 2064, out. 2000.

SOUZA, L. S.; COGO, N. P.; VIEIRA, S. R. Variabilidade de propriedades físicas e químicas do solo em um pomar cítrico. Revista Brasileira de Ciência do Solo, Campinas, v. 21, n. 3, p. 1-10, 1997.

VIEIRA, S. R. Variabilidade espacial de argila, silte e atributos químicos em uma parcela experimental de um latossolo roxo de Campinas (SP). Bragantia, Campinas, v. 56, n. 1, p. 1-17, 1997.

VIEIRA, S. R.; HATFIELD, J. L.; NIELSEN, D. R.; BIGGAR, J. W. Geoestatistical theory and application to variability of some agronomical properties. Hilgardia, Berkeley, v. 51, n. 3, p. 1-75, 1983. 